\title{
Comparative Endoscopic Anatomic Description of the Mitral Valvular Complex: a Cadaveric Study
}

\author{
Tamas Ruttkay ${ }^{1,2}$ Gabor Baksa ${ }^{2}$ Julia Gotte ${ }^{1}$ Tibor Glasz $^{3}$ Lajos Patonay ${ }^{2}$ Zoltan Galajda ${ }^{4}$ \\ Nicolas Doll ${ }^{1}$ Markus Czesla ${ }^{1}$
}

${ }^{1}$ Department of Cardiac Surgery, Sana Cardiac Surgery Stuttgart $\mathrm{GmbH}$, Stuttgart, Germany

2 Department of Anatomy, Histology and Embryology, Laboratory for

Address for correspondence Nicolas Doll, MD, PhD, Department of Cardiac Surgery, Sana Cardiac Surgery Stuttgart GmbH, Herdweg 2, Applied and Clinical Anatomy, Semmelweis University, Budapest, 70174 Stuttgart, Germany (e-mail: nicolas.doll@sana.de).

Hungary

${ }^{3}$ 2nd Department of Pathology, Semmelweis University, Budapest, Hungary

4 Department of Cardiac and Vascular Surgery, University of Debrecen, Debrecen, Hungary

Thorac Cardiovasc Surg 2015;63:231-237.

\begin{abstract}
Keywords

- anatomy

- mitral valve surgery

- minimally invasive surgery

Background We compared the aortic, left atrial, and apical approaches to visualize the mitral valve with the goal to investigate the endoscopic anatomy and give exact step-bystep descriptions of these views.

Materials and Methods The mitral valvular complex of human cadaveric fresh hearts was investigated from three approaches using 0,30 , and 70 degrees rigid endoscopic optics. In 30 cases after the removal of the hearts, the endoscopes were introduced directly into the aortic root through an aortotomy, left atrium through a standard atriotomy, and apex of the heart through a transmural incision. In 10 cases, the in situ visualization was performed using standard surgical approaches, such as partial upper ministernotomy, right and left minithoracotomy. The investigation was performed first with the mitral valve open, then the left ventricle was filled with saline, and the valve was closed by clamping the aorta.

Results For the visualization of ventricular surfaces of the mitral leaflets and the subvalvular apparatus, the apical approach was most optimal. The aortic approach had limitations at the posterior leaflet. Using the atrial approach, we did not obtain any direct visual information about the subvalvular apparatus with the valve closed. The atrial surfaces of the leaflets were best visible using both the atrial and apical approaches with the mitral valve open. In the case of a closed valve, the apical approach did not allow for an investigation of the atrial surfaces. The aortic approach was useful to visualize the atrial surface of the posterior leaflet with an opened valve.

Conclusion In mitral valve repairs through the left atrium, an additional aortic or apical view could be useful to obtain functional information about the subvalvular apparatus by the sealing probe.
\end{abstract}

received

August 5, 2013

accepted

October 1, 2013

published online

January 13, 2014 (c) 2015 Georg Thieme Verlag KG

Stuttgart · New York
DOI http://dx.doi.org/

10.1055/s-0033-1359322. ISSN 0171-6425. 


\section{Introduction}

The increasing number of minimally invasive mitral valve repair methods motivated the investigation of endoscopic mitral valve visualization and its anatomic description. Multiple approaches have been used to describe the anatomy of this region. The left atrial method, which is the most commonly method used in clinical practice, has generated extensive literature describing both the anatomic findings as well as the mitral repair techniques. ${ }^{1-7}$ The limitations of this approach have initiated research to provide a better visualization of the subvalvular apparatus. Experimental in situ animal procedures have been described for transapical intracardiac imaging ${ }^{8,9}$ and mitral repairs. ${ }^{10,11}$ In vitro animal ${ }^{12}$ and human ${ }^{13}$ studies have examined the functional anatomic parameters of the heart valves in the beating heart with endoscopic optics through the great vessels. To provide a more precise and detailed endoscopic anatomic description of the mitral valvular complex, we investigated the anatomy from multiple directions in cadaveric hearts. We selected the conventional left atrial, the developing apical, and the novel aortic approaches to visualize the valve and its related structures in the unfilled heart and the saline-filled left ventricle. The anatomic landmarks provided offer valuable help for the cardiac surgeon to identify the structures promptly using endoscopic optics. We outline exact stepby-step descriptions of different views from multiple directions and compare their advantages in mitral valve repair procedures.

\section{Materials and Methods}

The endoscopic views of the mitral valvular complex were examined in 40 human cadaveric fresh hearts ( 22 female, 18 male; aged 49-88 years). All hearts were obtained and dissected at the Department of Anatomy, Histology and Embryology (Semmelweis University, Budapest, Hungary), and no ethical approval for this study was necessary. Thirty of them were removed from the chest before the investigation and 10 were observed in situ, within the thorax. The mitral valves did not demonstrate any pathological findings.

\section{Exposure and Imaging}

In this study, we have used three approaches for the endoscopic examination: the aortic approach through the aortic valve, the atrial approach through the left atrium, and the apical approach through the apex of the heart. Three different endoscopes were used: 0,30 , and 70 degrees $4 \mathrm{~mm}$ rigid optics (Aesculap, Tuttlingen, Germany, PE 484A). The following exposures of the heart were performed for in situ investigation.

\section{Aortic Approach}

After standard partial upper sternotomy and pericardiotomy, a 2-cm-long transversal incision was performed at the aortic root $1 \mathrm{~cm}$ superiorly to the aortic valve. Using a sucker and saline injection, the blood was washed out from the left heart. The $4 \mathrm{~mm}$ endoscopes were inserted through the leaflets of the aortic valve under direct visual control. After clamping the aorta, the left ventricle was injected with saline solution under pressure using a silicon tube.

\section{Atrial Approach}

After standard right anterolateral minithoracotomy in the third intercostal space and pericardial incision, a 3-cm-long transmural incision was made on the left atrium $1 \mathrm{~cm}$ anteriorly and parallel to the line between the right superior and inferior pulmonary veins. An atrial retractor was placed to have an optimal exposure of the mitral valve. The structures of the mitral valvular complex were investigated with the rigid, $4 \mathrm{~mm}$ endoscopic optics. The left ventricle was filled with saline solution.

\section{Apical Approach}

After standard left anterolateral minithoracotomy in the fifth intercostal space, the pericardium was opened and a $1-\mathrm{cm}$ long transmural incision was performed on the apex of the heart lateral to the left anterior descending coronary artery branch. The apex of the heart was pulled with patch sutures to the skin incision. Introducing the rigid, $4 \mathrm{~mm}$ endoscopes into the left ventricle, the structures within it were inspected. Then the left ventricle was injected with saline solution under pressure using a simple silicon tube.

The heart and the aforementioned layers of the chest were closed after each approaches with running sutures, reconstructing the original situation. This step gave us the possibility for a real in situ anatomic investigation of the next approaches. The filling of the left ventricle was performed in all cases with the aorta clamped in the interest of optimal pressure.

In the other 30 cases, the hearts were removed from the thorax performing median sternotomy. The aorta and pulmonary artery were resected $2 \mathrm{~cm}$ superiorly to the valve commissures. The superior and inferior caval veins and each pulmonary vein were transected from the right and left atrium leaving a $1-\mathrm{cm}$ cuff. Before endoscopic examination, each heart was rinsed in saline solution. The removed hearts were suspended by using a plastic cylinder $(20 \mathrm{~cm}$ long and $12 \mathrm{~cm}$ diameter) with $5 \mathrm{~mm}$ holes on it, $2 \mathrm{~cm}$ apart. Making stitches around the apex of the heart, the mitral annulus, and the left atrium, the natural forms of the atrial and ventricular cavity were simulated. The endoscopic optics were inserted through the three aforementioned incisions: aortic approach, atrial approach, and apical approach. The anatomical investigation of the mitral valvular complex was performed first without filling of the left ventricle, and then it was injected under pressure with saline resulting in closure of the mitral leaflets. During this step, the aorta was clamped. All views of the mitral valvular complex were documented with color photographs using a Canon 5D camera with a Canon endoscopic adapter and the rigid, $4 \mathrm{~mm}$ endoscopic optics $(0,30$, 70 degrees, Aesculap).

\section{Results}

For the sake of plasticity of the heart and great vessels, determination of parameters for the introduction of the 
Table 1 Visibility of the mitral valvular complex and the aortic valve using the investigated approaches

\begin{tabular}{|c|c|c|c|c|c|c|}
\hline \multirow[t]{2}{*}{ Visible structures } & \multicolumn{2}{|c|}{ Aortic view } & \multicolumn{2}{|c|}{ Atrial view } & \multicolumn{2}{|c|}{ Apical view } \\
\hline & $\begin{array}{l}\text { Unfilled } \\
\text { heart }\end{array}$ & $\begin{array}{l}\text { Filled left } \\
\text { ventricle }\end{array}$ & Unfilled heart & $\begin{array}{l}\text { Filled left } \\
\text { ventricle }\end{array}$ & Unfilled heart & $\begin{array}{l}\text { Filled left } \\
\text { ventricle }\end{array}$ \\
\hline \multicolumn{7}{|l|}{ Mitral valvular complex } \\
\hline Line of mitral annulus & $+1-$ & $+1-$ & + & + & ++ & ++ \\
\hline $\begin{array}{l}\text { Atrial surface of } \\
\text { the leaflets }\end{array}$ & + & - & ++ & ++ & ++ & - \\
\hline $\begin{array}{l}\text { Ventricular surface } \\
\text { of the leaflets }\end{array}$ & + & + & - & - & ++ & ++ \\
\hline Commissures & - & - & ++ & ++ & ++ & ++ \\
\hline Chords & ++ & ++ & + & - & ++ & ++ \\
\hline Papillary muscles & ++ & ++ & + & - & ++ & ++ \\
\hline \multicolumn{7}{|l|}{ Aortic valve } \\
\hline Aortic surface of the cusps & ++ & + & - & - & - & - \\
\hline $\begin{array}{l}\text { Ventricular surface of } \\
\text { the cusps }\end{array}$ & + & + & - & - & ++ & ++ \\
\hline
\end{tabular}

endoscopes was limited. The aforementioned exact anatomic locations for the introduction were clear and by using fixed landmarks, the momentary position of the optic could be determined. Variations in the introducing angle and the accurate deepness were variable and caused by different measures of the individual hearts investigated. - Table 1 summarizes the visibility of the mitral valvular complex and the aortic valve using the investigated approaches.

\section{Aortic View}

In this approach, we selected the 70 degrees optic based on the excellent visibility of the anterior and posterior mitral leaflets and the subvalvular apparatus. Three different views were described at three different depths with the mitral valve open without filling and one view by closed valve after filling the left ventricle with saline under pressure.

\section{Unfilled Heart, Aortic View 1}

For the first view, the introduced 70 degrees endoscope was directed toward the anterior leaflet of the mitral valve situated the commissure between the posterior and left coronary aortic cusps at 12 o'clock. From the commissure downward straggling structures were identified as the ventricular surfaces of the posterior noncoronary aortic cusp on the left side and the left coronary cusp on the right side. Underneath, the aortomitral continuity was located forming a convex line. The entire ventricular surface of the anterior mitral leaflet could be seen under the aforementioned line. From left to right, the A3, A2, and A1 scallops were distinguished. The inserting part of the anterior marginal chords and the two thicker strut chords appeared on that surface of the anterior mitral leaflet (-Fig. 1a).

\section{Unfilled Heart, Aortic View 2}

For the second view, introducing the endoscopic optic deeper, the upper structure was the line of the aortomitral continuity.
Under this line, one could see the ventricular surface of the anterior mitral leaflet with the insertions of the anterior marginal and strut chords. In addition, the whole length and the origins of the chords were visible on this view. The two bands of chords were convergent on the left and the right half of the picture and originated from the papillary muscles. One could recognize the apical part of the posterior and anterior papillary muscles at 7 o'clock and 5 o'clock, respectively (-Fig. 1b, c).

\section{Unfilled Heart, Aortic View 3}

On the third view, as the endoscope was moved through the two chords running to the anterior leaflet of the mitral valve, the posterior leaflet became visible. The horizontal line of mitral annulus was situated at the upper part of this view. Under the line of the annulus, the atrial surface of the posterior mitral leaflet appeared. From left to right, the P3, $\mathrm{P} 2$, and $\mathrm{P} 1$ scallops could be seen. The chords running to the posterior leaflet converged downward to the posterior papillary muscle on the left side and to the anterior papillary muscle on the right side. The posterior marginal chords were visible in their whole length from the papillary muscles to the margin of the posterior leaflet. However, the insertions of intermediate and basal chords on the ventricular surface were hidden. The P3 and P1 scallops could be examined closely rotating the optic at 30 degrees to both directions around its longitudinal axis, but the posteromedial and anterolateral commissures could not be investigated yet ( - Fig. 1d).

\section{Filled Left Ventricle, Aortic View 4}

After filling the left ventricle with saline, the closed mitral valve could be inspected from the aortic valve. In this case, similarly to the open valve, the ventricular surface of the anterior mitral leaflet, as well as, from left to right, the A3, A2, and $A 1$ scallops were represented on the upper half of the view. All the anterior marginal and strut chords ran with the 


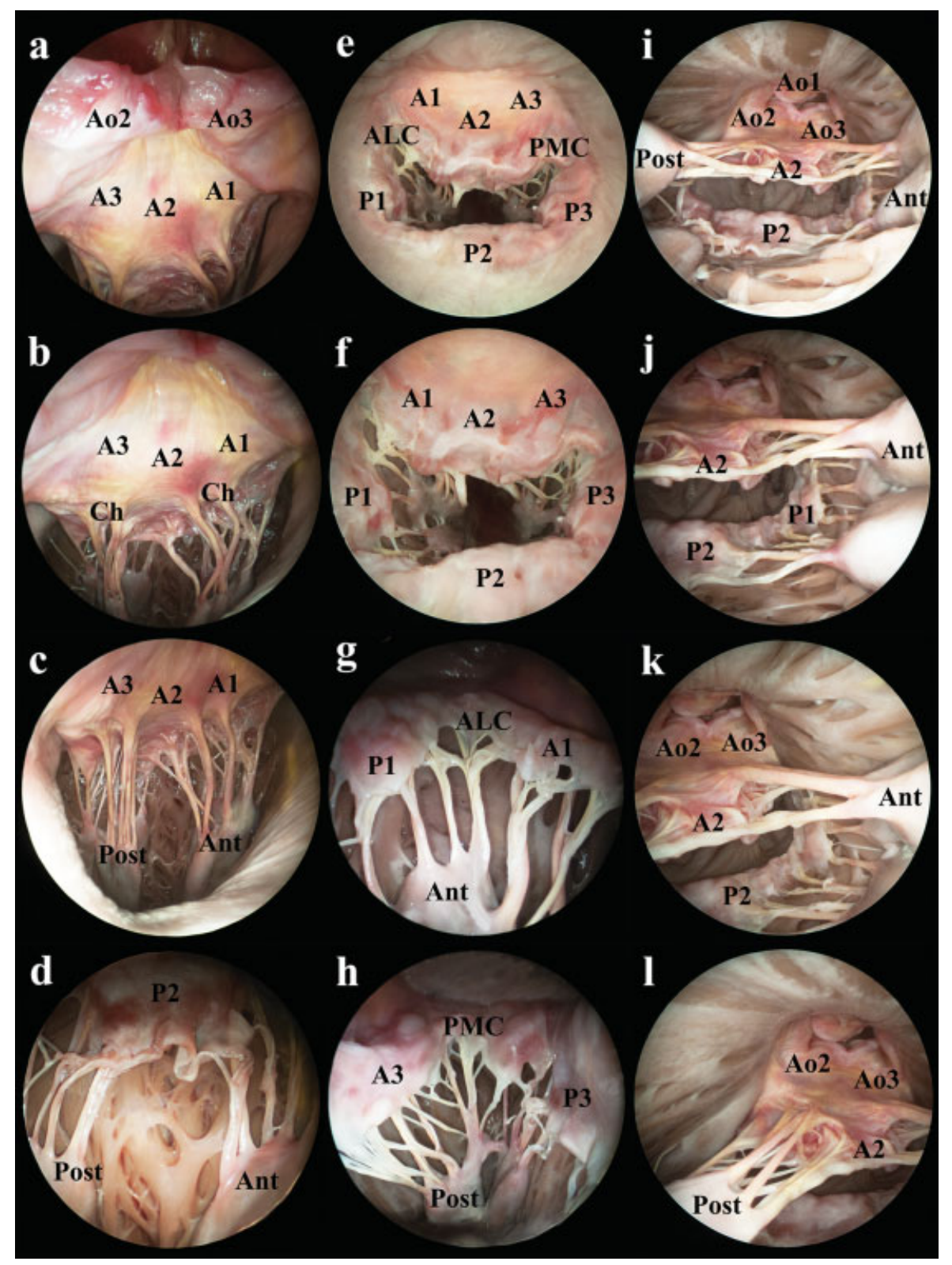

Fig. 1 The endoscopic view of the mitral valve introduced (a-d) a 70 degrees rigid endoscope through the aortic valve, (e, $f$ ) a 0 and $(g, h)$ a 70 degrees endoscope through the left atrium, and $(i-I)$ a 0 degrees endoscope through the apex of the heart without saline filling of the left ventricle. Ao1: right coronary cusp of the aortic valve; Ao2: posterior noncoronary cusp of the aortic valve; Ao3: left coronary cusp of the aortic valve; A1, A2, A3: A1, A2, and A3 scallops of the anterior mitral leaflet; P1, P2, P3: P1, P2, and P3 scallops of the posterior mitral leaflet; ALC: anterolateral commissure; PMC: posteromedial commissure; Ant: anterior papillary muscle; Post: posterior papillary muscle; Ch: tendinous chords.

posterior marginal, intermediate, and basal chords, as two bands, from the papillary muscles to the leaflets. The chords were in a suspended state because of the closing of the mitral valve. The posterior papillary muscle was visible on the left side of the view at 7 o'clock and the anterior papillary muscle on the right side at 5 o'clock. The commissures and the coaptation line could not be seen directly. The entire distended subvalvular apparatus, as well as the closing function

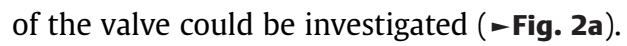

\section{Atrial View}

During examination from the atrial approach, the 0 and 30 degrees endoscopes gave an optimal view of the mitral leaf- lets. To investigate the subvalvular apparatus with the mitral valve opened, the 70-degree optic was more helpful. After filling the left ventricle with saline, the closed mitral valve could be examined optimally using the 0 and 30 degrees endoscopes. These two optics gave about the same views.

\section{Unfilled Heart, Atrial View 1}

Using the 0 degrees optic, the ring of the mitral annulus filled the view with the leaflets and the subvalvular apparatus. Positioning the A2 scallop at 12 o'clock, the anterior leaflet was visible between the commissures, under the line of the aortomitral continuity. The anterolateral commissure was situated on the left upper side of the view, at 10 o'clock. 


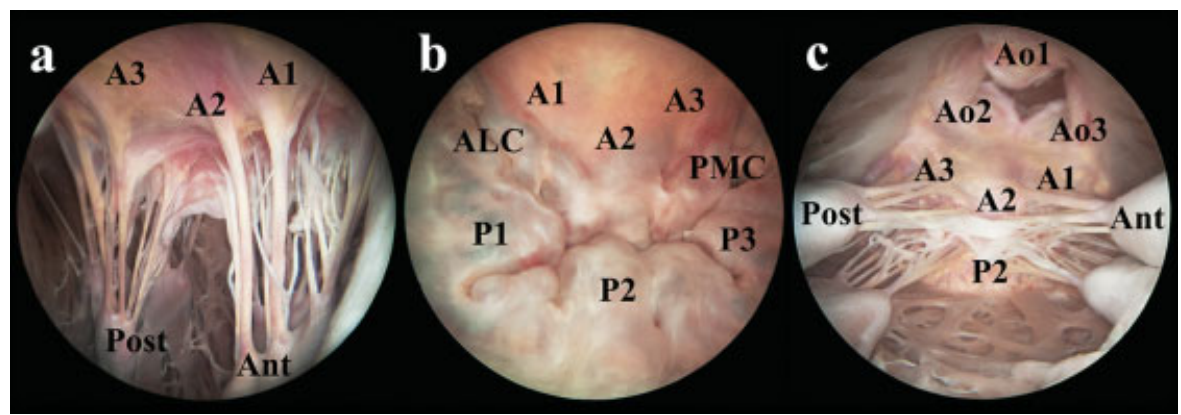

Fig. 2 The endoscopic view of the mitral valve introduced (a) a 70 degrees rigid endoscope through the aortic valve, (b) a 0 degrees endoscope through the left atrium, and (c) a 0 degrees endoscope through the apex of the heart with saline filling of the left ventricle. Ao1: right coronary cusp of the aortic valve; Ao2: posterior noncoronary cusp of the aortic valve; Ao3: left coronary cusp of the aortic valve; A1, A2, A3: A1, A2, and A3 scallops of the anterior mitral leaflet; P1, P2, P3: P1, P2, and P3 scallops of the posterior mitral leaflet; ALC: anterolateral commissure; PMC: posteromedial commissure; Ant: anterior papillary muscle; Post: posterior papillary muscle.

From left to right, the atrial surfaces of the $\mathrm{A} 1, \mathrm{~A} 2$, and $\mathrm{A} 3$ scallops were visualized, terminated by the posterolateral commissure at 2 o'clock. In the orifice, between the anterior and posterior mitral leaflets, the subvalvular apparatus was visible. The anterior and posterior marginal chords were inserted on the free margins of the leaflets. Conversely, the visibility of strut, posterior intermediate and basal chords were limited. These chords could be followed from their origins on the papillary muscles, but their insertions on the ventricular surface of the leaflets could not be seen. The apical region of the anterior papillary muscle could be found on the left side and the posterior papillary muscle on the right side of the view. Under the mitral orifice, the atrial surface of the posterior leaflet was positioned, from left to right, the P1, P2, and P3 scallops. The standard and deviant clefts of the posterior leaflet were located between the scallops, as small fissures (-Fig. 1e, f).

\section{Unfilled Heart, Atrial View 2}

Introducing the 70 degrees endoscope into the orifice of the mitral valve, the structures of the subvalvular apparatus could be examined in richer detail. The anterolateral commissure was positioned at 12 o'clock. The P1, P2 scallops of the posterior leaflet, as well as the A1, A2 scallop of the anterior leaflet could be followed straggling downward from the high middle located commissure, with P1, P2 on the left side of the view and A1, A2 on the right side. The chords, originating from the anterior papillary muscle, were seen in the central zone of the view, encircled by the atrial surfaces of the leaflets. The commissural chords were located on the main vertical axis of the view, surrounded by the posterior marginal chords on the left and the anterior marginal chords on the right. Although the marginal chords could be visualized in their whole length, the visualization of the insertions of the posterior intermediate and basal chords was limited. The anterior papillary muscle was situated in the middle on the bottom of the view (-Fig. 19).

\section{Unfilled Heart, Atrial View 3}

After a 90-degree rightward rotation of the endoscope around its longitudinal axis, the atrial surface of the anterior mitral leaflet became visible, as well as its upper border, the line of aortomitral continuity. On the surface of the tongueshaped anterior leaflet, all three scallops, such as, from left to right, A1, A2, and A3 could be characterized. Under the free margin of the leaflet, the downward straggling anterior chords could be investigated.

\section{Unfilled Heart, Atrial View 4}

Rotating the optic by another 90 degrees, the posteromedial commissure could be seen, offering a similar view as previously described by the anterolateral commissure, just with opposite directions. The posteromedial commissure was situated at 12 o'clock. The atrial surfaces of the leaflets were downward straggling, with the A3 scallop on the left side of the view and the P3 scallop on the right side. In the middle zone of the view, the aforementioned commissural, anterior marginal, posterior marginal, intermediate, and basal chords could be visualized. The posterior papillary muscle was positioned in the middle on the bottom part of the view (-Fig. 1h).

\section{Unfilled Heart, Atrial View 5}

After another 90 degrees right rotation of the endoscope around its longitudinal axis, the atrial surface of the posterior leaflet filled in the field of vision with its subvalvular apparatus. Under the line of the mitral annulus, all three scallops of the leaflet could be seen, with the P3 scallop on the left side, the P2 scallop in the middle, and the P1 scallop on the right side of the view. Under the free margin of the leaflet, few details of the subvalvular apparatus, especially the posterior marginal chords were found.

\section{Filled Left Ventricle, Atrial View 6}

After filling the left ventricle with saline, the atrial surface of the closed mitral valve could be optimally visualized, but we did not get any direct visual information about the chords and papillary muscles. On the upper side of the view, the A1, A2, and $\mathrm{A} 3$ scallops of the anterior leaflet were situated between the anterolateral commissure on the left and the posteromedial commissure on the right. Under the semilunar coaptation line, the P1, P2, and P3 scallops of the posterior leaflet could be characterized, as well as the bordering standard and deviant clefts on its surface ( - Fig. $\mathbf{2 b}$ ). 


\section{Apical View}

After testing the apical approach with different endoscopic optics, the 0 degrees endoscope was found optimal for visualization of the whole mitral valvular complex, with both opened and closed mitral valve. In the investigation of the smaller details, the 30 and 70 degrees optics proved themselves to be useful too. The description of the complex was given step by step in the left ventricle starting from the apex, with and without saline filling.

\section{Unfilled Heart, Apical View 1}

Introducing the 0 degrees endoscope through the apical incision, the trabeculae of the left ventricle were the first to appear in the field of vision. Directing the anterior wall of the left ventricle in the upper part of the view, the interventricular septum was situated on the left, the left marginal wall on the right, and the posterior wall on the bottom part of the view. The different anatomical variations of the trabeculae were filled in the foreground of the view as myocardial bridges. The mitral valve was suspected in the deepness.

\section{Unfilled Heart, Apical View 2}

Examining the mitral valve deeper in the left ventricle, all structures of the complex could be seen on the same view. The ventricular surface of the aortic valve was positioned at 12 o'clock. The right coronary cusp of the aortic valve was located on the top, with the posterior cusp on the left side and the left coronary cusp on the right side under it. The line of the aortomitral continuity was situated exactly under the aortic valve. As the left angle of the mitral orifice, the posteromedial commissure could be visualized under the aforementioned anatomical structures. From left to right, the ventricular surfaces of the $A 3, A 2$, and A1 scallops of the anterior mitral leaflet were found, ending with the anterolateral commissure. The anterior marginal chords and the two strut chords could be identified as they reached the free margin and the ventricular surface of the anterior leaflet, starting from their origin on the posterior and anterior papillary muscles. The posterior papillary muscle was located on the left side of the orifice and the anterior papillary muscle on the right side. As the lower margin of the orifice, the posterior leaflet was to be seen. The whole length of the posterior marginal, intermediate, and basal chords could be visualized, inserting the P3 scallop on the left, P2 scallop in the middle, and P1 scallop on the right. Both the atrial and ventricular surfaces of the leaflets could not be investigated from the apex. Encircling the mitral orifice, the line of the mitral annulus could be followed, exactly in the angle of the posterior leaflet and the ventricular wall ( - Fig. 1i-I).

\section{Filled Left Ventricle, Apical View 3}

After filling the left ventricle with saline under pressure, the optimal visual examination of the closed mitral leaflets and the subvalvular apparatus was possible with the 0 degrees endoscopic optic. The view was just the same as without saline filling. Under the three leaflets of the aortic valve and the aortomitral continuity, from left to right, the posteromedial commissure, the A3, A2, A1 scallops of the anterior leaflet and the anterolateral commissure were situated. Under the coaptation line, similarly from left to right, the P3, P2, and P1 scallops of the posterior leaflet, along with their bordering clefts were positioned. The posterior papillary muscle was located on the left side and the anterior papillary muscle on the right side of the coaptation line. All the distended anterior and posterior chords could be visualized in their whole length, originating from the papillary muscles and the free wall of the left ventricle, inserting on the free margins and the ventricular surfaces of the leaflets. The line of the mitral annulus was located in the angle of the posterior leaflet and the wall of the left ventricle (-Fig. $2 \mathbf{c}$ ).

Generally all mitral valves could be visualized perfectly using any of the described methods without any significant difficulties. The technically easiest approach was the atrial. Using the aortic and apical approaches, some anatomical variations such as pathological findings influenced the investigation. We refer to two important aspects: first, the left ventricular hypertrophy and second, the length of the chords. Left ventricular hypertrophy resulting in smaller cavity measures limited free movement of the optics and thus the visualization of all details in case of the aortic and especially the apical approaches. Such conditions were found in 14 (35\%) of the 40 investigated hearts. In case of the aortic approach, relatively short chords resulted in difficulties when the endoscope was introduced between the chords of the anterior mitral leaflet to observe the posterior leaflet, occurring in 16 (40\%) hearts.

\section{Discussion}

Extensive literature on minimally invasive mitral valve repair using the left atrial approach has been published in the last couple of years. ${ }^{1-7}$ Publications focusing on anatomic aspects as well as methodical issues reported overall good results of this procedure. The left atrial approach offers optimal visualization of the mitral valve leaflets. The subvalvular apparatus can only be visualized when the valve is opened. At the sealing probe, surgeons do not become any direct visual information about the status of the papillary muscles and tendinous chords. To solve this problem in other previous publications, an impressive transapical in vivo endoscopic imaging of the mitral and tricuspid valves has been described. ${ }^{8,10,11}$ In these cases, a cardiopulmonary bypass circuit supported the systemic organ perfusion and a separate transparent fluid circuit in the left heart allowed for visualization of intracardiac structures. Anatomic structures were only described in general terms, but these publications outlined a novel approach and method for future valve repair procedures under beating heart conditions. The endoscopic investigation through blood was described by a beating heart animal model. ${ }^{9}$ The top of the endoscope introduced into the left ventricle was covered with a convex Plexiglas and the tissues in front of the cardioscope could be seen. Other animal and human studies were using beating heart models to investigate the movement of intracardiac structures by explanted hearts. ${ }^{12,13}$ An endoscopic optic was introduced through the great vessels. Dynamic images of the motion of different valves during contraction and relaxation phases of 
the cardiac cycle will lead to a more profound understanding of cardiac physiology, pathology, and pathophysiology. Under echocardiography guidance, it was possible to implant neochords in off-pump animal studies. ${ }^{14}$ Using a complex, apically introduced system after fixing on the leaflet margin, the length of the neochords was adjustable from the outside. Transapical mitral valved stent implantations were described in native valves under transesophageal echocardiography guidance in animal experiments, ${ }^{15}$ as well as clinical valvein-valve implantations by deteriorated bioprotheses. ${ }^{16}$ Both the neochord implantations and mitral valve stent implantations outline the real possibility and importance of transapical procedures in mitral valve repair.

The novel concept in our study consisted of investigating three-dimensional anatomic structural confirmations by analyzing and comparing various different approaches. We noticed that all of the directions used revealed new visual information on the examined structures. The description of those three different entries studied in this publication highlights their individual advantages and drawbacks in detail. A thorough and complex knowledge of the anatomy of the mitral valve could help the surgeon in understanding and teaching various mitral valve repair techniques. The atrial endoscopic view is an optimal approach for annuloplasty ring replacement and leaflet resection. For artificial chord replacement, we prefer a complementary view near the conventional atrial approach. The aortic or apical view could help at the implantation of chords as well as the functional investigation of the subvalvular apparatus by filling the left ventricle. For edge-to-edge repair techniques, the apical view using biportal endoscopic control is preferable since it allows for perfect visibility of the coaptation line. The in vivo intraoperative transapical introduction of an additional endoscope requires a separate left minithoracotomy in standard minimally invasive mitral repairs or the displacement of the heart including distortion of the anatomy in median sternotomy cases. The introduction of an additional optic through the aortic valve is possible by just performing a small aortotomy in median sternotomy approaches. The surgeon has to decide in each case whether perfect visualization or minimal access surgery is more important.

Further development of the currently only experimentally used transapical view could turn this approach into the most useful view since it allows for a perfect visualization of the entire mitral valvular complex throughout the surgical repair.

\section{Acknowledgments}

The authors are grateful to Prof. Dr. Andras Csillag (Department of Anatomy, Histology and Embryology, Semmelweis University, Budapest) for allowing this study. The authors also thank Dr. Miklos Sarvari (Institute of Experimental Medicine, Hungarian Academy of Sciences, Budapest, Hungary) and Dr. Miklos Toth (Department of Otorhinolaryngology, Paracelsus Medical University Salzburg, Austria) for reading the article.

\section{References}

1 Chitwood WR Jr, Elbeery JR, Chapman WH, et al. Video-assisted minimally invasive mitral valve surgery: the "micro-mitral" operation. J Thorac Cardiovasc Surg 1997;113(2):413-414

2 Chitwood WR Jr, Elbeery JR, Moran JF. Minimally invasive mitral valve repair using transthoracic aortic occlusion. Ann Thorac Surg 1997;63(5):1477-1479

3 Casselman FP, Van Slycke S, Dom H, Lambrechts DL, Vermeulen Y, Vanermen H. Endoscopic mitral valve repair: feasible, reproducible, and durable. J Thorac Cardiovasc Surg 2003;125(2):273-282

4 Seeburger J, Borger MA, Falk V, et al. Minimal invasive mitral valve repair for mitral regurgitation: results of 1339 consecutive patients. Eur J Cardiothorac Surg 2008;34(4):760-765

5 Smith JM, Stein H. Endoscopic placement of multiple artificial chordae with robotic assistance and nitinol clip fixation. J Thorac Cardiovasc Surg 2008;135(3):610-614

6 Vollroth M, Seeburger J, Garbade J, et al. Minimally invasive mitral valve surgery is a very safe procedure with very low rates of conversion to full sternotomy. Eur J Cardiothorac Surg 2012;42(1): e13-e15, e16

7 Quill JL, Hill AJ, Laske TG, Alfieri O, Iaizzo PA. Mitral leaflet anatomy revisited. J Thorac Cardiovasc Surg 2009;137(5):1077-1081

8 Mihaljevic T, Ootaki Y, Robertson JO, et al. Beating heart cardioscopy: a platform for real-time, intracardiac imaging. Ann Thorac Surg 2008;85(3):1061-1065

9 Padala M, Jimenez JH, Yoganathan AP, Chin A, Thourani VH. Transapical beating heart cardioscopy technique for off-pump visualization of heart valves. J Thorac Cardiovasc Surg 2012; 144(1):231-234

10 Shiose A, Takaseya T, Fumoto H, et al. Cardioscopy-guided surgery: intracardiac mitral and tricuspid valve repair under direct visualization in the beating heart. J Thorac Cardiovasc Surg 2011;142(1): 199-202

11 Horai T, Fukamachi K, Fumoto H, et al. Direct endoscopy-guided mitral valve repair in the beating heart: an acute animal study. Innovations (Phila) 2011;6(2):122-125

12 Chinchoy E, Soule CL, Houlton AJ, et al. Isolated four-chamber working swine heart model. Ann Thorac Surg 2000;70(5): 1607-1614

13 Hill AJ, Laske TG, Coles JA Jr, et al. In vitro studies of human hearts. Ann Thorac Surg 2005;79(1):168-177

14 Seeburger J, Leontjev S, Neumuth M, et al. Trans-apical beatingheart implantation of neo-chordae to mitral valve leaflets: results of an acute animal study. Eur J Cardiothorac Surg 2012;41(1): 173-176, discussion 176

15 Lutter G, Quaden R, lino K, et al. Mitral valved stent implantation. Eur J Cardiothorac Surg 2010;38(3):350-355

16 Wilbring M, Alexiou K, Tugtekin SM, et al. Transapical transcatheter valve-in-valve implantation for deteriorated mitral valve bioprostheses. Ann Thorac Surg 2013;95(1):111-117 